\title{
Pinning Synchronization of Complex Dynamical Networks with Variable-Delayed Coupling by Periodically Intermittent Control
}

\author{
Ranran Cheng $\mathbb{D}^{1},{ }^{1}$ Xiaoyong Tian, ${ }^{1}$ Mingshu Peng, ${ }^{2}$ and Jinchen $\mathbf{Y u}^{3}$ \\ ${ }^{1}$ Department of Science, North China Institute of Science and Technology, Yanjiao 065201, China \\ ${ }^{2}$ Department of Mathematics, Beijing Jiao Tong University, Beijing 100044, China \\ ${ }^{3}$ Department of Science, Shandong Jiao Tong University, Jinan 250357, China \\ Correspondence should be addressed to Ranran Cheng; rrcheng@ncist.edu.cn
}

Received 18 December 2019; Revised 15 February 2020; Accepted 13 March 2020; Published 14 April 2020

Guest Editor: Vikas Kumar Mishra

Copyright $\odot 2020$ Ranran Cheng et al. This is an open access article distributed under the Creative Commons Attribution License, which permits unrestricted use, distribution, and reproduction in any medium, provided the original work is properly cited.

This paper studied the adaptive pinning synchronization in complex networks with variable-delay coupling via periodically intermittent control. Theoretical analysis is included by means of Lyapunov functions and linear matrix inequalities (LMI) to make all nodes reach complete synchronization. Moreover, the synchronization criteria do not impose any restriction on the size of time delay. Numerical examples including the regular, Watts-Strogatz and scale-free BA random topological architecture are provided to illustrate the importance of our theoretical analysis.

\section{Introduction}

Complex dynamical networks have been intensively studied $[1,2]$ over the last few years due to their potential applications in various fields of the real world, such as in the biological systems, scientific citation web, social networks, and electrical power grids and thus became an important part of our daily life.

Since Pecora and Carroll [3] found chaos synchronization in 1990, it has become a focal research topic recently. Due to the synchronization not only can well explain many natural phenomena observed, such as synchronized firefly flashing and swarming of fishes, but also has many practical potential applications such as image processing, the operation of unmanned aerial vehicle, and secure communication. However, the complex network cannot synchronize by itself, so many control methods have been developed, such as linear and nonlinear feedback control, time-delay feedback control, sliding mode control [4], adaptive control [5], pinning control [6], impulsive control [7,8], and intermittent control [9-12]. Especially, discontinuous feedback controls, including impulsive control [13] and intermittent control, have been attracting much attention since they are practically and easily implemented in some engineering domains. However, the intermittent control is different from the impulsive control because impulsive control is activated only at some isolated moments, while the intermittent control has a nonzero control width.

Intermittent control was first introduced to control chaos systems by Zochowski [9] in 2000. It is more efficient when the system output is measured intermittently rather than continuously. There are some novel synchronization criteria in complex networks with delay or nondelay coupling by intermittent control. Li et al. [14] studied the inner synchronization of delayed nonlinear chaotic systems by intermittent control. Further, Mei et al. [10] used diver and response systems to produce the finite-time synchronization for the complex systems without delay. In [12], the authors investigated the synchronization problem of stochastic perturbed complex systems with time-varying delays. On the contrary, complex networks have a large number of nodes in the real world, and it is usually impractical to control a 
complex network by adding the controllers to all nodes. To reduce the number of controlled nodes, pinning control, in which controllers are only applied to partial nodes, is introduced [15-20]. In [21], the authors discussed the synchronization for coupled dynamical networks with mixed delays and uncertain parameters using pinning control and intermittent control. By means of intermittent control, the authors [22] studied finite-time synchronization for a class of reaction-diffusion neural networks by small domains on their spatial boundaries, associated with an interaction graph. Li et al. [23] and Xu et al. [24]discussed the synchronization problem of general complex networks with fractional-order dynamical networks by periodically intermittent pinning control.

Moreover, in $[11,15]$, the authors investigated the synchronization of complex networks with delays by pinning periodically intermittent control; especially, they assumed that the control width needs to be larger than the time delay or the time-varying delays should be differentiable, and their derivatives are less than 1. Motivated by the above discussions, we removed these constraints in our results. In this paper, we give the complex systems with both timevarying delays and nondelay couplings, and using pinning control and periodically intermittent control methods, some novel criteria for pinning synchronization are derived. Numerical examples including the regular, Watts-Strogatz and scale-free BA random topological architecture are provided to illustrate the importance of our theoretical analysis.

The rest of the paper is organized as follows: in Section 2, we propose a general complex dynamical network model; some preliminaries and lemmas are given. In Section 3, some pinning adaptive synchronization criteria for the general complex dynamical networks with delay coupling are given. Numerical examples are given in Section 4. Finally, we draw our conclusion in Section5.

\section{Preliminaries and Mathematical Models}

Consider a generally controlled complex delayed dynamical system consisting of $N$ nodes, with each node being of $n$ dimensions, which is described by

$$
\begin{aligned}
\dot{x}_{i}(t)= & A x_{i}(t)+f\left(x_{i}(t)\right)+c_{0} \sum_{j=1}^{N} b_{i j} \Gamma x_{j}(t) \\
& +c_{1} \sum_{j=1}^{N} \bar{b}_{i j} \Gamma x_{j}(t-\tau(t)),
\end{aligned}
$$

where $1 \leq i \leq N, \quad x_{i}(t)=\left(x_{i 1}(t), \ldots, x_{i n}(t)\right)^{T} \in \mathbb{R}^{n}$ is the state variable of node $i, A \in \mathbb{R}^{n \times n}$ is a given constant matrix, and $f: \mathbb{R}^{n} \longrightarrow \mathbb{R}^{n}$ is a continuously differentiable function describing the nonlinear dynamics of the single node. Here, $c_{0}$ and $c_{1}$ are two parameters of the nondelay and delay coupling strength, respectively, $\Gamma=\left(\gamma_{i j}\right) \in \mathbb{R}^{n \times n}$ is an inner- coupling matrix, and $\tau(t)$ is the coupled delay and bounded by a known constant, i.e., $0<\tau(t)<\tau$. $B=\left(b_{i j}\right)_{N \times N}$ and $\bar{B}=$ $\left(\bar{b}_{i j}\right)_{N \times N}$ represent the adjacency configuration of the network with the nondelay and delay couplings, respectively; if there is a link from node $i$ to node $j$, then $b_{i j}>0$ (or $\bar{b}_{i j}>0$ ) $(j \neq i)$; otherwise, $b_{i j}=0 \quad$ (or $\left.\bar{b}_{i j}=0\right)$. Moreover, $b_{i i}=-\sum_{j \neq i} b_{i j}$ and $\bar{b}_{i i}=-\sum_{j \neq i} \bar{b}_{i j}$.

As we know, the complex network cannot synchronize by itself; then, we add the adaptive controller $v_{i}(t)$ as follows:

$$
\begin{aligned}
\dot{x}_{i}(t)= & A x_{i}(t)+f\left(x_{i}(t)\right)+c_{0} \sum_{j=1}^{N} b_{i j} \Gamma x_{j}(t) \\
& +c_{1} \sum_{j=1}^{N} \bar{b}_{i j} \Gamma x_{j}(t-\tau(t))+v_{i}(t) .
\end{aligned}
$$

Hereafter, let $s(t)=s\left(t ; t_{0}, x_{0}\right) \in R^{m}$ be a solution of the node system $\dot{x}(t)=f(x(t))$. Then, $s(t)$ is a synchronous solution of controlled complex delayed dynamical system (2). Note that $s(t)$ may be an equilibrium point, a periodic orbit, or a chaotic attractor.

Define error vectors as

$$
e_{i}(t)=x_{i}(t)-s(t), \quad 1 \leq i \leq N .
$$

According to system (2), the error system is described by

$$
\begin{aligned}
\dot{e}_{i}(t)= & A e_{i}+f\left(x_{i}(t)\right)-f\left(y_{i}(t)\right)+c_{0} \sum_{j=1}^{N} b_{i j} \Gamma e_{j}(t) \\
& +c_{1} \sum_{j=1}^{N} \bar{b}_{i j} \Gamma e_{j}(t-\tau(t))+v_{i}(t),
\end{aligned}
$$

where $1 \leq i \leq N$.

Lemma 1 (Schur complement, see [25]). The linear matrix inequality (LMI) is as follows:

$$
\left(\begin{array}{cc}
A(x) & B(x) \\
B^{T}(x) & C(x)
\end{array}\right)>0,
$$

where $A(x)=A^{T}(x), C(x)=C^{T}(x)$ is equivalent to one of the following conditions:

$$
\begin{aligned}
& \text { (1) } A(x)>0, C(x)-B^{T}(x) A^{-1}(x) B(x)>0 \\
& \text { (2) } C(x)>0, A(x)-B(x) C^{-1}(x) B^{T}(x)>0
\end{aligned}
$$

Lemma 2 (see [26]). Let $X$ and $Y$ be arbitrary $n$-dimensional real vectors, $K$ be a positive definite matrix, and $P \in \mathbb{R}^{m \times m}$. Then, the following matrix inequality holds:

$$
2 X^{T} P Y \leq X^{T} P K^{-1} X+Y^{T} K Y .
$$

\section{Main Results}

In the following, assume that $\Gamma \neq 0$ and $\|\Gamma\|_{2}=\gamma>0$. Denote $\rho_{i i}$ as the minimum eigenvalue of the matrix $\left(\Gamma+\Gamma^{T}\right) / 2$. Let 
$\widehat{B}^{s}=\left(\widehat{B}+\widehat{B}^{T}\right) / 2$, and its eigenvalues are expressed as $\lambda_{1} \geq \lambda_{2} \geq \ldots \geq \lambda_{N}$, where $\widehat{B}$ is a modifying matrix of $B$ via replacing the diagonal elements $b_{i i}$ by $\left(\rho_{\min } / \gamma\right) b_{i i}$. Note that, generally, $\widehat{B}$ does not possess the property of zero row sums.

To realize the network synchronization, the controllers $v_{i}$ should guide the error vectors $e_{i}(t)$ to approach zero as $t$ goes to infinity as

$$
\lim _{t \longrightarrow \infty}\left\|e_{i}(t)_{2}\right\|=0,1 \leq i \leq N .
$$

Choose the adaptive controllers as follows:

$$
v_{i}(t)= \begin{cases}-p_{i}(t) e_{i}(t), 1 \leq i \leq l, & t \in[m T,(m+h) T), \\ 0, \quad l+1 \leq i \leq N, & t \in[m T,(m+h) T, \\ 0, \quad 1 \leq i \leq N, & t \in[(m+h) T,(m+1) T),\end{cases}
$$

where $\dot{p}_{i}(t)=\exp \left\{a_{1} t\right\} q_{i} e_{i}^{T}(t) e_{i}(t), q_{i}$, and $a_{1}$ are positive constants. $T>0$ denotes the control period, $0<h<1$ is the rate of control duration called control rate, and $m=0,1,2, \ldots$.

Theorem 1. Suppose that $\left\|f\left(x_{i}\right)-f\left(y_{i}\right)\right\|_{2} \leq \beta\left\|e_{i}\right\|_{2}$. If there exists a positive constant $a_{1}, a_{2}$, and $k$ such that

$$
\begin{array}{r}
\left(\alpha+\beta+\frac{a_{1}}{2}+\frac{c_{1} \gamma\left[(N-1) \bar{b}_{1}+k \bar{b}_{2}\right]}{2 k}\right) I_{N}+c_{0} \gamma \widehat{B}^{s}-D<0, \\
\alpha+\beta-\frac{a_{2}-a_{1}}{2}+\frac{c_{1} \gamma\left[(N-1) \bar{b}_{1}+k \bar{b}_{2}\right]}{2 k}+c_{0} \gamma \lambda_{1}<0, \\
\xi-a_{2}(1-h)>0,
\end{array}
$$

where $\|A\|_{2}=\alpha, \quad D=\operatorname{diag}(\underbrace{p, \ldots p}_{l}, \underbrace{0, \ldots 0}_{N-l}), \quad p>0$, $q=c_{1}\left[(N-1) k \bar{b}_{1}+\bar{b}_{2} \gamma\right], \quad \bar{b}_{1}=\max \left\{\bar{b}_{i j}, j \neq i\right\}, \quad a_{2}>a_{1}>q$, $\bar{b}_{2}=\max \left\{\bar{b}_{i i}\right\}$, and $\xi>0$ is the smallest real root of the equation $-a_{1}+\xi+q \exp (\xi \tau)=0$, then the synchronized manifold $\sim(s(t), s(t), \ldots, s(t))^{T}$ of controlled complex delayed dynamical system (2) is globally asymptotically stable under periodical intermittent controllers (8).

Proof. Construct the candidate Lyapunov function as follows:

$$
V(t)=\frac{1}{2} \sum_{i=1}^{N} e_{i}^{T}(t) e_{i}(t)+\frac{1}{2} \sum_{i=1}^{l} \exp \left(-a_{1} t\right) \frac{\left(p_{i}(t)-p\right)^{2}}{q_{i}},
$$

and when $m T \leq t \leq m T+h$, calculating the time derivative of $V(t)$ along the trajectories of (4), one has

$$
\begin{aligned}
\dot{V}(t)= & \sum_{i=1}^{N} e_{i}^{T}(t) e_{i}(t)+\sum_{i=1}^{l} \exp \left(-a_{1} t\right) \frac{\left(p_{i}(t)-p\right) p_{i}(t)}{q_{i}}-\frac{a_{1}}{2} \sum_{i=1}^{l} \exp \left(-a_{1} t\right) \frac{\left(p_{i}(t)-p\right)^{2}}{q_{i}} \\
= & \sum_{i=1}^{N} e_{i}^{T}(t)\left(A e_{i}+f\left(x_{i}\right)-f(s)\right)+c_{0} \sum_{i=1}^{N} \sum_{j=1}^{N} b_{i j} e_{i}^{T}(t) \Gamma e_{j}(t)+c_{1} \sum_{i=1}^{N} \sum_{j=1}^{N} \bar{b}_{i j} e_{i}^{T} \Gamma e_{j}(t-\tau(t)) \\
& -\sum_{i=1}^{l} p_{i}(t) e_{i}^{T}(t) e_{i}(t)+\sum_{i=1}^{l} \exp \left(-a_{1} t\right) \frac{\left(p_{i}(t)-p\right) \dot{p}_{i}(t)}{q_{i}}-\frac{a_{1}}{2} \sum_{i=1}^{l} \exp \left(-a_{1} t\right) \frac{\left(p_{i}(t)-p\right)^{2}}{q_{i}} \\
\leq & (\alpha+\beta) \sum_{i=1}^{N} e_{i}^{T}(t) e_{i}(t)+c_{0} \sum_{i=1}^{N} \sum_{j=1}^{N} b_{i j} e_{i}^{T} \Gamma e_{j}(t)+c_{1} \sum_{i=1}^{N} \sum_{j=1}^{N} \bar{b}_{i j} e_{i}^{T} \Gamma e_{j}(t-\tau(t)) \\
& -\sum_{i=1}^{l} p e_{i}^{T}(t) e_{i}(t)-\frac{a_{1}}{2} \sum_{i=1}^{l} \exp \left(-a_{1} t\right) \frac{\left(p_{i}(t)-p\right)^{2}}{q_{i}} .
\end{aligned}
$$


From Lemma 2, one can find

$$
\begin{aligned}
& 2 \sum_{i=1}^{N} \sum_{j=1}^{N} \bar{b}_{i j} e_{i}^{T} \Gamma e_{j}(t-\tau(t)) \leq \sum_{i=1}^{N} \sum_{j=1, j \neq i}^{N} \bar{b}_{i j}\left[e_{i}^{T}(t) \Gamma K^{-1} e_{i}(t)+e_{j}(t-\tau(t))^{T} K e_{j}(t-\tau(t))\right] \\
&+2 \sum_{i=1}^{N}\left|\bar{b}_{i i}\right| \gamma\left\|e_{i}(t)\right\|_{2}\left\|e_{i}(t-\tau)\right\|_{2} \\
& \leq \sum_{i=1}^{N} \sum_{j=1, j \neq i}^{N}\left[\frac{\bar{b}_{1} \gamma}{k} e_{i}^{T}(t) e_{i}(t)+k \bar{b}_{1} e_{j}^{T}(t-\tau(t)) e_{j}(t-\tau(t))\right] \\
&+\sum_{i=1}^{N}\left|\bar{b}_{i i}\right| \gamma\left[e_{i}^{T}(t) e_{i}^{T}(t)+e_{i}^{T}(t-\tau(t)) e_{i}^{T}(t-\tau(t))\right] \\
& c_{0} \sum_{i=1}^{N} \sum_{j=1}^{N} b_{i j} e_{i}^{T} \Gamma e_{j}(t) \leq c_{0} \sum_{i=1}^{N} \sum_{j=1, j \neq i}^{N} \gamma b_{i j}\left\|e_{i}(t)\right\|_{2}\left\|e_{j}(t)\right\| \|_{2}+c_{0} \sum_{i=1}^{N} b_{i i} \rho_{\min } e_{i}^{T}(t) e_{i}(t), \\
&=\left[\frac{(N-1) \bar{b}_{1} \gamma}{k}+\bar{b}_{2} \gamma\right] \sum_{i=1}^{N} e_{i}^{T}(t) e_{i}(t)+\left[(N-1) k \bar{b}_{1}+\bar{b}_{2} \gamma\right] \sum_{i=1}^{N} e_{i}^{T}(t-\tau(t)) e_{i}(t-\tau(t)),
\end{aligned}
$$

where $\|K\|_{2}=k,\|\Gamma\|_{2}=\gamma, \bar{b}_{1}=\max \left\{\bar{b}_{i j}, j \neq i\right\}, \bar{b}_{2}=\max \left\{\bar{b}_{i i}\right\}$, and $\rho_{i i}$ is the minimum eigenvalue of the matrix $\left(\Gamma+\Gamma^{T}\right) / 2$. It follows from (12) and (13) that

$$
\begin{aligned}
\dot{V}(t) \leq & e^{T}(t)\left[\left(\alpha+\beta+\frac{c_{1} \gamma\left[(N-1) \bar{b}_{1}+k \bar{b}_{2}\right]}{2 k}\right) I_{N}+c_{0} \gamma \widehat{B}^{s}-D\right] e(t) \\
& +\frac{c_{1}\left[(N-1) k \bar{b}_{1}+\bar{b}_{2} \gamma\right]}{2} e^{T}(t-\tau(t)) e(t-\tau(t))-\frac{a_{1}}{2} \sum_{i=1}^{l} \exp \left(-a_{1} t\right) \frac{\left(p_{i}(t)-p\right)^{2}}{q_{i}} \\
\leq & e^{T}(t)\left[\left(\alpha+\beta+\frac{a_{1}}{2}+\frac{c_{1} \gamma\left[(N-1) \bar{b}_{1}+k \bar{b}_{2}\right]}{2 k}\right) I_{N}+c_{0} \gamma \widehat{B}^{s}-D\right] e(t) \\
& +\frac{q}{2} e^{T}(t-\tau(t)) e(t-\tau(t))-\frac{a_{1}}{2}\left[\sum_{i=1}^{N} e^{T}(t) e(t)+\sum_{i=1}^{l} \exp \left(-a_{1} t\right) \frac{\left(p_{i}(t)-p\right)^{2}}{q_{i}}\right] \\
\leq & -a_{1} V(t)+q V(t-\tau(t)),
\end{aligned}
$$


where $e(t)=\left(\left\|e_{1}(t)\right\|_{2},\left\|e_{2}(t)\right\|_{2}, \ldots\left\|e_{\underline{N}}(t)\right\|_{2}{ }_{2}{ }^{T}, \quad D=\operatorname{diag} \quad\right.$ When $m T+h \leq t \leq(m+1) T$, $(\underbrace{p, \ldots p}_{l}, \underbrace{0, \ldots 0}_{N-l})$, and $q=c_{1}\left[(N-1) k \bar{b}_{1}+\bar{b}_{2} \gamma\right]$.

$$
\begin{aligned}
\dot{V}(t)= & \sum_{i=1}^{N} e_{i}^{T}(t) \dot{e}_{i}(t)-\frac{a_{1}}{2} \sum_{i=1}^{l} \exp \left(-a_{1} t\right) \frac{\left(p_{i}(t)-p\right)^{2}}{q_{i}} \\
= & \sum_{i=1}^{N} e_{i}^{T}(t)\left(A e_{i}+f\left(x_{i}\right)-f(s)\right)+c_{0} \sum_{i=1}^{N} \sum_{j=1}^{N} b_{i j} e_{i}^{T}(t) \Gamma e_{j}(t)+c_{1} \sum_{i=1}^{N} \sum_{j=1}^{N} \bar{b}_{i j} e_{i}^{T} \Gamma e_{j}(t-\tau(t)) \\
& -\frac{a_{1}}{2} \sum_{i=1}^{l} \exp \left(-a_{1} t\right) \frac{\left(p_{i}(t)-p\right)^{2}}{q_{i}} \\
\leq & (\alpha+\beta) \sum_{i=1}^{N} e_{i}^{T}(t) e_{i}(t)+c_{0} \sum_{i=1}^{N} \sum_{j=1}^{N} b_{i j} e_{i}^{T} \Gamma e_{j}(t)+c_{1} \sum_{i=1}^{N} \sum_{j=1}^{N} \bar{b}_{i j} e_{i}^{T} \Gamma e_{j}(t-\tau(t)) \\
& -\frac{a_{1}}{2} \sum_{i=1}^{l} \exp \left(-a_{1} t\right) \frac{\left(p_{i}(t)-p\right)^{2}}{q_{i}} \\
\leq & e^{T}(t)\left[\left(\alpha+\beta+\frac{c_{1} \gamma\left[(N-1) \bar{b}_{1}+k \bar{b}_{2}\right]}{2 k}\right) I_{N}+c_{0} \gamma \widehat{B}^{s}\right] e(t) \\
\leq & \left(a_{2}-a_{1}\right) V(t)+q V(t-\tau(t)) . \\
& +\frac{a_{2}-a_{1}\left[\sum_{i=1}^{N} e^{T}(t) e(t)+\sum_{i=1}^{l} \exp \left(-a_{1} t\right) \frac{\left(p_{i}(t)-p\right)^{2}}{q_{i}}\right]}{\leq} e^{T}(t)\left[\left(\alpha+\beta-\frac{a_{2}-a_{1}}{2}+\frac{c_{1} \gamma\left[(N-1) \bar{b}_{1}+k \bar{b}_{2}\right]}{2 k}\right) I_{N}+c_{0} \gamma \widehat{B}^{s}\right] e(t)+\frac{q}{2} e^{T}(t-\tau(t)) e(t-\tau(t)) \\
& +\frac{q}{2} e^{T}(t-\tau) e(t-\tau(t))-\frac{a_{1}}{2} \sum_{i=1}^{l} \exp \left(-a_{1} t\right) \frac{\left(p_{i}(t)-p\right)^{2}}{q_{i}} \\
&
\end{aligned}
$$

Namely, we have

$$
\left\{\begin{array}{c}
\dot{V}(t) \leq-a_{1} V(t)+q V(t-\tau(t)), \quad t \in[m T,(m+h) T), \\
\dot{V}(t) \leq\left(a_{2}-a_{1}\right) V(t)+q V(t-\tau(t)), t \in[(m+h) T, \\
(m+1) T) .
\end{array}\right.
$$

In the following, we will prove that

$$
V(t) \leq \sup _{-\tau \leq \theta \leq 0} V(\theta) \exp \left\{-\left[\xi-a_{2}(1-h)\right] t\right\}, \quad t \geq 0 .
$$

Denote $g(\xi)=-a_{1}+\xi+q \exp (\xi \tau)$; as $a_{1}>q>0$, we have $g(0)<0, g(\infty)>0$, and $g^{\prime}(\xi)>0$. Using the continuity and the monotonicity of the function, $g(\xi)=0$ has a unique positive solution $\xi>0$. Let $M=\sup _{-\tau \leq \theta \leq 0} V(\theta)$ and $W(t)=\exp \{\xi t\} V(t)$.

$Q_{1}(t)=W(t)-\epsilon M, \epsilon>1$ is a constant. Obviously,

$$
Q_{1}(t)<0, \quad \text { for all } t \in[-\tau, 0] .
$$

Next, we will prove that

$$
Q_{1}(t)<0, \quad \text { for all } t \in[0, h T] .
$$

Otherwise, there exists $t_{1} \in[0, h T]$ such that

$Q_{1}\left(t_{1}\right)=0, \dot{Q}_{1}\left(t_{1}\right) \geq 0$, and $Q_{1}(t)<0,-\tau \leq t<t_{1}$.

Using (16) and (20), one obtains 


$$
\begin{aligned}
\dot{Q}_{1}\left(t_{1}\right) \quad & =\xi \exp \left\{\xi t_{1}\right\} V\left(t_{1}\right)+\exp \{\xi t\} \dot{V}\left(t_{1}\right) \\
& \leq\left(\xi-a_{1}\right) W(t)+q \exp \left\{\xi \tau\left(t_{1}\right)\right\} W\left(t_{1}-\tau\left(t_{1}\right)\right) \\
& <\left(-a_{1}+\xi+q \exp (\xi \tau)\right) \varepsilon M=0 .
\end{aligned}
$$

This leads to a contradiction with (20); hence, (19) holds. Now, we prove that, for $t \in[h T, T]$,

$$
Q_{2}(t)=W(t)-\varepsilon M \exp \left\{a_{2}(t-h T)\right\}<0 .
$$

Otherwise, there exists $t_{2} \in[h T, T]$ such that

$Q_{2}\left(t_{2}\right)=0, \dot{Q}_{2}\left(t_{2}\right) \geq 0$, and $Q_{2}(t)<0, \quad h T \leq t<t_{2}$.

Then,

$$
\begin{aligned}
\dot{Q}_{2}\left(t_{2}\right)= & \xi W\left(t_{2}\right)+\exp \left\{\xi t_{2}\right\} \dot{V}\left(t_{2}\right)-a_{2} \varepsilon M \exp \left\{a_{1}(t-h T)\right\} \\
\leq & \left(\xi+a_{2}-a_{1}\right) W(t)+q \exp \left\{\xi \tau\left(t_{2}\right)\right\} W\left(t_{2}-\tau\left(t_{2}\right)\right) \\
& -a_{2} \varepsilon M \exp \left\{a_{2}(t-h T)\right\} .
\end{aligned}
$$

For $0<\tau(t)<\tau$, if $h T \leq t_{2}-\tau\left(t_{2}\right)<t_{2}$, then from (23), one has

$$
W\left(t_{2}-\tau\left(t_{2}\right)\right)<\varepsilon M \exp \left\{a_{1}\left(t_{2}-h T\right)\right\},
$$

and if $-\tau \leq t_{2}-\tau\left(t_{2}\right)<h T$, from (19), one obtains

$$
W\left(t_{2}-\tau\left(t_{2}\right)\right)<\varepsilon M<\varepsilon M \exp \left\{a_{2}\left(t_{2}-h T\right)\right\} .
$$

So,

$$
\dot{Q}_{2}\left(t_{2}\right)<\left(-a_{1}+\xi+q \exp (\xi \tau)\right) \varepsilon M \exp \left\{a_{2}\left(t_{2}-h T\right)\right\}=0 \text {. }
$$

This leads to a contradiction with (23); hence, (22) holds. According (19) and (22), one obtains

$$
W(t)<\varepsilon M \exp \left\{a_{2}(1-h) T\right\} \text {, for all } t \in[-\tau, T) \text {. }
$$

Similarly, we can prove that

$$
\begin{array}{ll}
W(t)<\varepsilon M \exp \left\{a_{2}(1-h) T\right\}, & t \in[T,(1+h) T), \\
W(t)<\varepsilon M \exp \left\{a_{2}(t-2 h) T\right\}, & t \in[(1+h) T, 2 T) .
\end{array}
$$

By induction, we can derive the following estimation of $W(t)$ for any integer $m$ :

$W(t)<\varepsilon M \exp \left\{a_{2} m(1-h) T\right\}, \quad t \in[m T,(m+h) T)$,

$W(t)<\varepsilon M \exp \left\{a_{2}(t-(m+1) h) T\right\}, \quad t \in[(m+h) T,(m+1) T)$.

Since for any $t \geq 0$, there exists a nonnegative integer $r$ such that $r T \leq t \leq(r+1) T$; then, we have

$$
\begin{gathered}
W(t)<\varepsilon M \exp \left\{a_{2} r(1-h) T\right\} \leq \varepsilon M \exp \left\{a_{2}(1-h) t\right\} \\
t \in[r T,(r+h) T) \\
W(t)<\varepsilon M \exp \left\{a_{2}(t-(r+1) h) T\right\} \leq \varepsilon M \exp \left\{a_{2}(1-h) t\right\} \\
t \in[(r+h) T,(r+1) T) .
\end{gathered}
$$

Let $\varepsilon \longrightarrow 1$, and from the definition of $W(t)$, one has

$$
V(t) \leq M \exp \left\{-\left[\xi-a_{2}(1-h)\right] t\right\} \text {. }
$$

As $V(t)=1 / 2 \sum_{i=1}^{N} e_{i}^{T}(t) e_{i}(t)+1 / 2 \sum_{i=1}^{l} \exp \left(-a_{1} t\right)\left(\left(p_{i}\right.\right.$ $\left.(t)-p)^{2} / q_{i}\right)$, one has

$$
\|e(t)\|_{2} \leq(2 M)^{(1 / 2)} \exp \left\{-\frac{\xi-a_{2}(1-h)}{2} t\right\} .
$$

In view of $\xi-a_{2}(1-h)>0$, we can draw the conclusion. The proof is thus completed.

Let

$$
\begin{gathered}
\eta=\alpha+\beta+\frac{a_{1}}{2}+\frac{c_{1} \gamma\left[(N-1) \bar{b}_{1}+k \bar{b}_{2}\right]}{2 k}, \\
M=\eta I_{N}+c_{0} \gamma \widehat{B}^{s}-D=\left(\begin{array}{cc}
Q-\widetilde{D} & G \\
G^{T} & \tilde{M}
\end{array}\right),
\end{gathered}
$$

where $\widetilde{D}=\operatorname{diag}(\underbrace{p, \ldots p}_{l}), \widetilde{M}$ is obtained by removing the $1,2, \ldots, l$ row-column pairs of matrix $M$, and $Q$ and $G$ are matrices with appropriate dimensions.

According to Lemma 1 , one can easily see that $M<0$ is equivalent to $\tilde{M}<0$ because of $Q-\widetilde{D}-G \widetilde{M}^{-1} G^{T}<0$ by choosing $p>\lambda_{\max }\left(Q-G \widetilde{M}^{-1} G^{T}\right)$, i.e., $M<0$ is equivalent to $\tilde{M}<0$ for sufficiently large $p$. Note that $\tilde{M}=\eta I_{N-l}+c \gamma \widehat{B}^{s}$, where $\widehat{B}_{i, j}^{s}=\widehat{B}_{(l+i),(l+j)}^{s}, i, j=1,2, \ldots, N-l$. Then, one can immediately get the following corollary:

Corollary 1. Suppose that $\left\|f\left(x_{i}\right)-f\left(y_{i}\right)\right\|_{2} \leq \beta\left\|e_{i}\right\|_{2}, \tau \leq h$ and $\tau \leq T-h$. If there exists a positive constant $a_{1}, a_{2}$, and $k$ such that

$$
\begin{aligned}
\bar{\lambda}_{l} & =\lambda_{\max }\left(\overline{\widehat{B}}^{s}\right)<-\frac{\eta}{c_{0} \gamma}, \\
& \alpha+\beta-\frac{a_{2}-a_{1}}{2}+\frac{c_{1} \gamma\left[(N-1) \bar{b}_{1}+k \bar{b}_{2}\right]}{2 k}+c_{0} \gamma \lambda_{1}<0, \\
& \xi-a_{2}(1-h)>0,
\end{aligned}
$$

where $\|A\|_{2}=\alpha, q=c_{1}\left[(N-1) k \bar{b}_{1}+\bar{b}_{2} \gamma\right], \bar{b}_{1}=\max \left\{\bar{b}_{i j}, j \neq\right.$ $i\}, a_{2}>a_{1}>q, \bar{b}_{2}=\max \left\{\bar{b}_{i i}\right\}$, and $\|\Gamma\|_{2}=\gamma, \xi>0$ is the smallest real root of the equation $-a_{1}+\xi+q \exp (\xi \tau)=0$, then the synchronized manifold $(\overbrace{s(t), s(t), \ldots, s(t)}^{N})^{T}$ of controlled complex delayed dynamical system (2) is globally asymptotically stable under periodical intermittent controllers (8).

Remark 1. The number of control nodes $l$ can be chosen properly to adjust the synchronization efficiency of networks. However, it is noted that $l$ by the theoretical prediction is only a sufficient condition but not a necessary one. In simulations, we will show that a small value of $l$ can also lead to synchronization.

\section{Numerical Simulation}

In this section, a numerical example is used to verify the effectiveness of the proposed network synchronization criteria.

Consider the Lorenz oscillator model described by the following equation: 


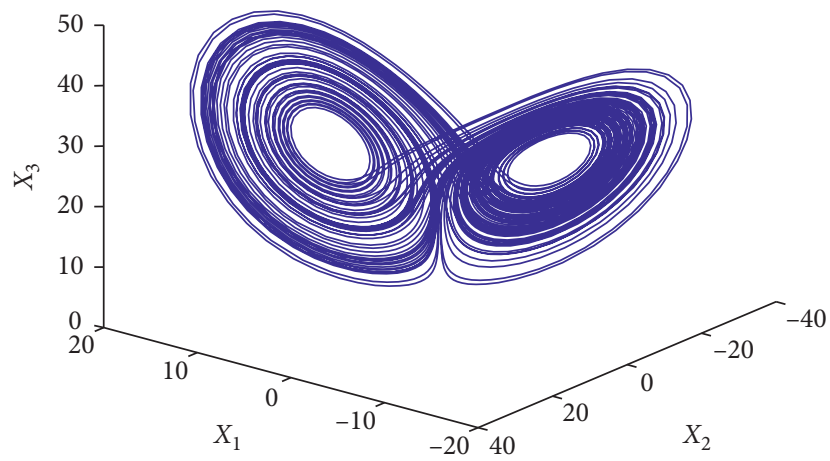

Figure 1: The chaotic attractor of the Lorenz system.

TABLE 1: Parameters in system (38) with different topological architecture.

\begin{tabular}{lccc}
\hline Parameter & Regular $p=0$ & Small-world $p=0.1$ & Scale-free \\
\hline $\bar{b}_{2}$ & 8 & 14 & 47 \\
$\lambda_{1}$ & 0.8561 & 0.9388 & 0.6623 \\
$q$ & 2.27601 & 2.3113 & 2.7439 \\
\hline
\end{tabular}

$$
\dot{x}_{i}(t)=A x_{i}+f\left(x_{i}\right)
$$

where $\quad x_{i}(t)=\left(x_{i 1}(t), x_{i 2}(t), x_{i 3}(t)\right)^{T} \in R^{3}, \quad f\left(x_{i}\right)=(0$, $\left.-x_{i 1}(t) x_{i 3}(t), x_{i 1}(t) x_{i 2}(t)\right)^{T}$, and

$$
A=\left(\begin{array}{ccc}
-10 & 10 & 0 \\
30 & -1 & 0 \\
0 & 0 & -3 / 8
\end{array}\right)
$$

It has been known that the Lorenz oscillators exhibit chaotic behavior, and Figure 1 shows it clearly.

Then, we consider controlled complex delayed dynamical system (2) consisting of 200 identical Lorenz systems, which are described by

$$
\begin{aligned}
\dot{x}_{i}(t)= & A x_{i}+f\left(x_{i}\right)+c_{0} \sum_{j=1}^{200} b_{i j} \Gamma x_{j}(t)+c_{1} \sum_{j=1}^{200} \bar{b}_{i j} \Gamma x_{j} \\
& \cdot(t-\tau)+v_{i}(t), \quad 1 \leq i \leq 200,
\end{aligned}
$$

where $\quad \Gamma=\operatorname{diag}\{1,1.2,1\} \quad$ and $B=\left(b_{i j}\right)_{200 \times 200} \quad$ and $\bar{B}=\left(\bar{b}_{i j}\right)_{200 \times 200}$ are symmetrically diffusive coupling matrices with $b_{i j}=0\left(\right.$ or $\left.\bar{b}_{i j}=0\right)$ or $1(j \neq i)$. Here, the coupling coefficient $c_{0}=30$.

As we know, Lorenz system is bounded. Here, we suppose $\left\|x_{i 1}\right\| \leq 29, \quad\left\|x_{i 2}\right\| \leq 29, \quad-1 \leq x_{i 3} \leq 57, \quad\left\|s_{1}\right\| \leq 29$, $\left\|s_{2}\right\| \leq 29,-1 \leq s_{3} \leq 57$, and $1 \leq i \leq 200$.

$$
\begin{aligned}
\left\|f\left(x_{i}\right)-f(s)\right\|_{2} & =\sqrt{\left(-x_{i 1} x_{i 3}+s_{1} s_{3}\right)^{2}+\left(x_{i 1} x_{i 2}-s_{1} s_{2}\right)^{2}} \\
& =\sqrt{\left(-x_{i 3} e_{i 1}-s_{1} e_{i 3}\right)^{2}+\left(x_{i 2} e_{i 1}+s_{1} e_{i 2}\right)^{2}} \\
& \leq 75.97\left\|\mathbf{e}_{i}\right\|_{2} .
\end{aligned}
$$

Suppose that the network structure of equation (38) obeys the regular $(p=0,2 \mathrm{~m}=8) /$ small-world $(p=0.3$, $2 \mathrm{~m}=8)[1] /$ scale-free (SF) [2] distribution, respectively. The number of the nodes $N=200$. Obviously, $\gamma=\|\Gamma\|_{2}=1.2$, $\alpha=\|A\|_{2}=31.91, \beta=75.97, \bar{b}_{1}=1$, and $\rho_{\min }=1$. Choosing the coupling coefficient $c_{0}=30, c_{1}=0.01, k=\sqrt{\gamma}, T=0.2$, $h=0.9$, and $\tau(t)=1+0.1|\sin t|<\tau=1.1$. By using the MATLAB LMI Toolbox and the corollary, one can obtain Table 1.

Please note that the average number of neighbours is the same between the small-world network with the connection probability $p=0.1$ and the regular network $(p=0)$, the number of neighbours becomes rand in the scale-free/smallworld topological structure, and $\max \left\{\bar{b}_{i i}\right\}$ can be estimated by the statistic method.

As $a_{1}-8<\xi<a_{1}-9$, then if we choose $a_{1}=45$ and $a_{2}=325$, it is easy to verify that the criteria in Theorem 1 are satisfied.

For the regular network $(p=0)$, one has $\eta=131.580$; from corollary, one can find $\lambda_{l^{*}}<-3.6531$. As $\lambda_{25}=-3.5984$ and $\lambda_{26}=-4.0041$, we can choose $l^{*}=26$. By numerical simulation, the synchronization error quickly tends to zero for $l=15<l^{*}$. This further indicates that $l>l^{*}$ is only a sufficient condition, and a small value of $l$ can also lead to synchronization.

The initial conditions of the numerical simulations are as follows: $\quad x_{i}(0)=(4+0.5 i, 5+0.5 i, 6+0.5 i)^{T}$ and $s(0)=$ $(4,5,6)^{T}$. Synchronous errors are shown in Figure 2 . And we choose different control periods, and the synchronous errors are shown in Figure 3. It is seen from the figures that smallworld or scale-free networks can reach complete synchronization by pin-controlling fewer nodes than regular systems. 

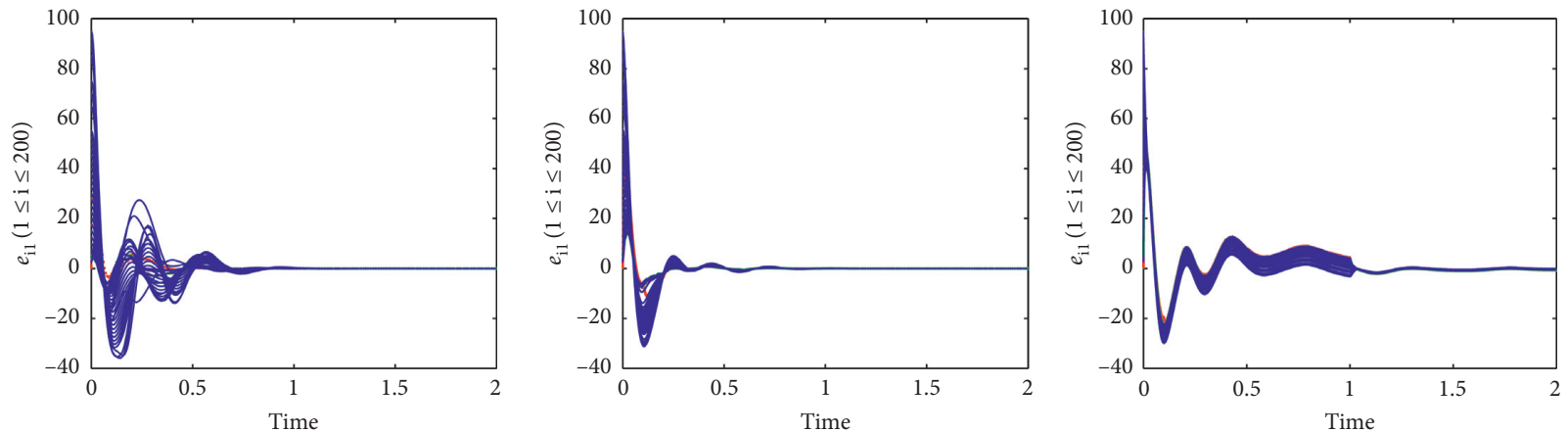

(a)
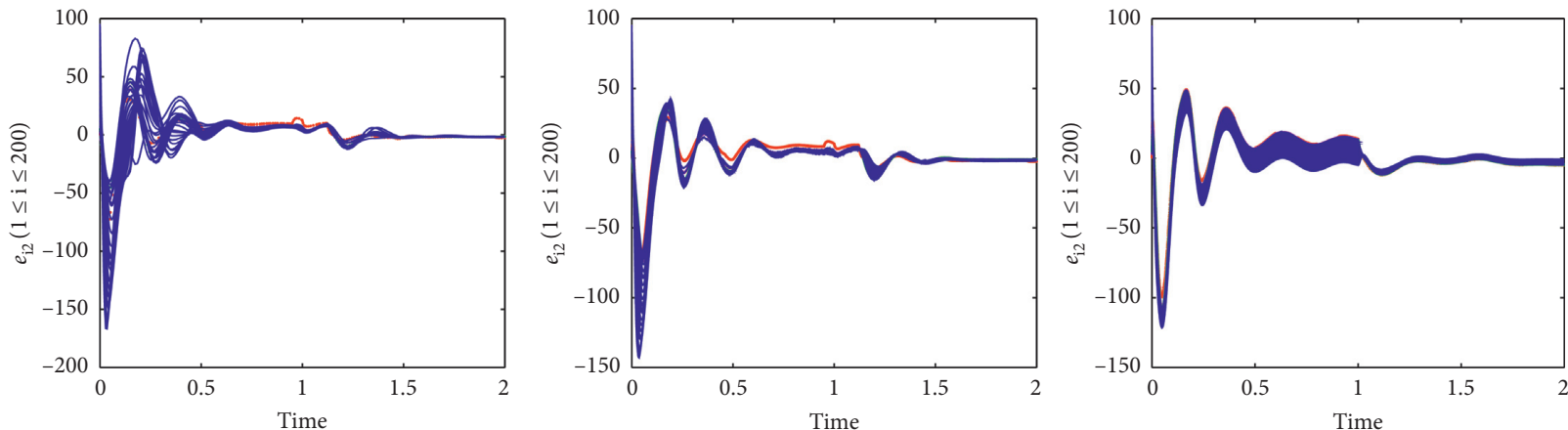

(b)
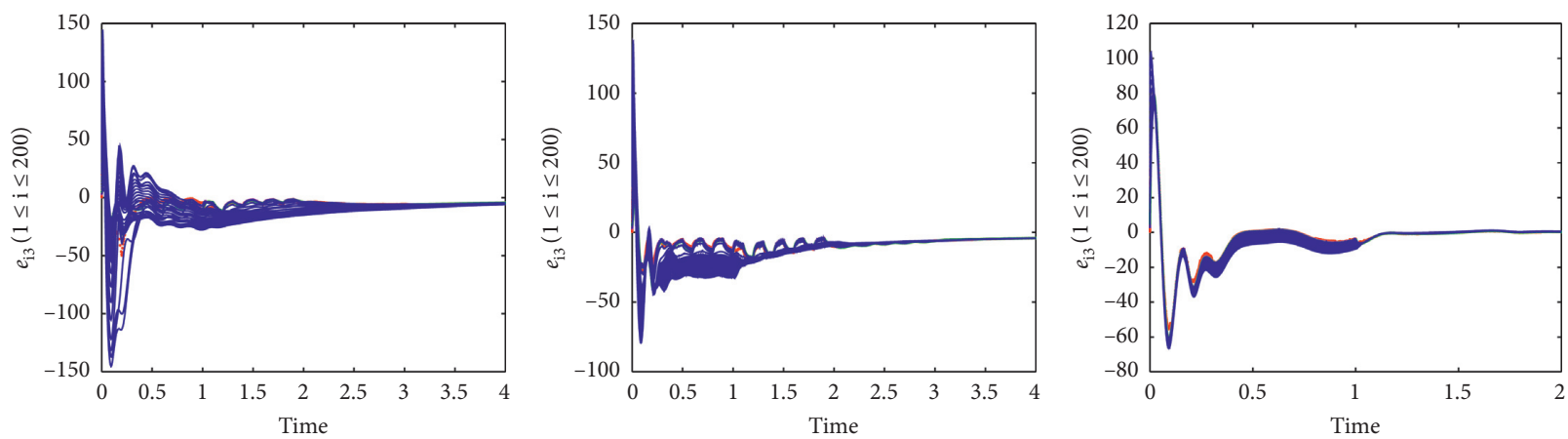

(c)

FiguRE 2: Synchronizability in system (38) with different topological architecture $(N=200)$ by pinning control: (a) regular networks with 8 neighbours, $l=15$, (b) WS small-world networks with the connection probability $p=0.1, l=5$, and (c) scale-free networks, $l=2$.

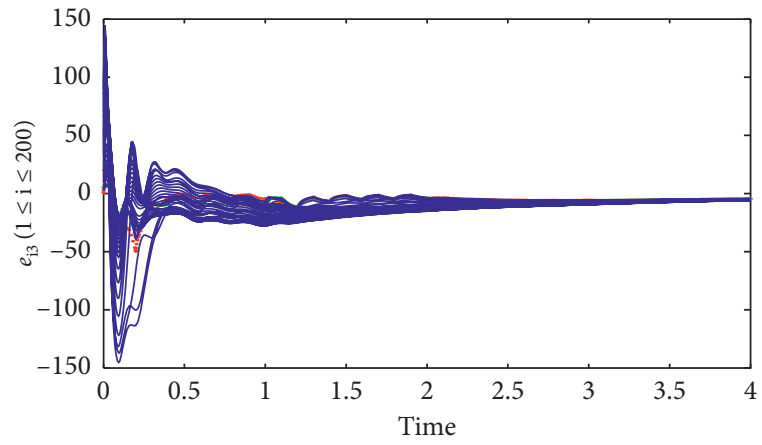

(a)

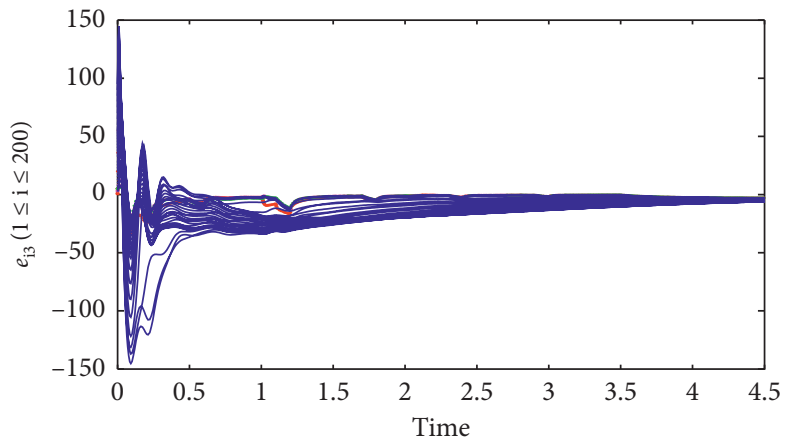

(b)

FIGURE 3: Synchronizability in system (38) with different periods: (a) $T=0.2$; (b) $T=0.5$. 


\section{Conclusions}

In this paper, we investigate adaptive pinning synchronization in complex delayed dynamical networks with timevarying delays by intermittent control. Based on the Lyapunov stability theory and chaos control method, several adaptive synchronization criteria are obtained. Our results show that the control width does not need to be larger than the time delays, and there is no restriction on the size of time delays. Moreover, we also find that small-world or scale-free networks can reach complete synchronization by pin-controlling fewer nodes than regular systems.

\section{Data Availability}

The data used to support the findings of this study are available from the corresponding author upon request.

\section{Conflicts of Interest}

The authors declare that they have no conflicts of interest.

\section{Acknowledgments}

This work was partially supported by the National Innovation Project (no. 201911104027), the Science and Technology Support Project of Langfang (no. 2016011052), the Fundamental Research Funds for the Central Universities of China (no. 3142017004), the School-Enterprise in Depth Cooperation Project (no. 1057), and the Teaching Reformation Project (no. HKJYGH201817).

\section{References}

[1] D. J. Watts and S. H. Strogatz, "Collective dynamics of "smallworld” networks," Nature, vol. 393, no. 6684, pp. 440-442, 1998.

[2] A.-L. Barabási and R. Albert, "Emergence of scaling in random networks," Science, vol. 286, no. 5439, pp. 509-512, 1999.

[3] L. M. Pecora and T. L. Carroll, "Synchronization in chaotic systems," Physical Review Letters, vol. 64, no. 8, pp. 821-824, 1990.

[4] H. Zhang, X. Y. Wang, and X. H. Lin, "Synchronization of complex-valued neural net- work with sliding mode control," Journal of the Franklin Institute, vol. 353, pp. 345-358, 2016.

[5] X. Wu, Y. Liu, and J. Zhou, "Pinning adaptive synchronization of general time-varying delayed and multi-linked networks with variable structures," Neurocomputing, vol. 147, pp. 492-499, 2015.

[6] R. Cheng, M. Peng, and W. Yu, "Pinning synchronization of delayed complex dynamical networks with nonlinear coupling," Physica A: Statistical Mechanics and Its Applications, vol. 413, pp. 426-431, 2014.

[7] Z. Guan, Z. Liu, and G. Feng, "Synchronization of complex dynamical networks with time-varying delays via impulsive distributed control," IEEE Transactions on Circuits and Systems, vol. 57, pp. 2182-2195, 2010.

[8] J. Lu, J. Kurths, and J. Cao, "Synchronization control for nonlinear stochastic dynamical networks: pinning impulsive strategy," IEEE Transactions on Neural Networks and Learning Systems, vol. 23, pp. 285-292, 2012.
[9] M. Zochowski, “Intermittent dynamical control," Physica D, vol. 145, pp. 181-190, 2000.

[10] J. Mei, M. Jiang, X. Wang, J. Han, and S. Wang, "Finite-time synchronization of drive-response systems via periodically intermittent adaptive control," Journal of the Franklin Institute, vol. 351, no. 5, pp. 2691-2710, 2014.

[11] W. Xia and J. Cao, "Pinning synchronization of delayed dynamical networks via periodically intermittent control," Chaos, vol. 19, 2009.

[12] J. Wang, J. Feng, C. Xu, and Y. Zhao, "Exponential synchronization of stochastic perturbed complex networks with time-varying delays via periodically intermittent pinning," Communications in Nonlinear Science and Numerical Simulation, vol. 18, no. 11, pp. 3146-3157, 2013.

[13] X. Yang and J. Cao, "Hybrid adaptive and impulsive synchronization of uncertain complex networks with delays and general uncertain perturbations," Applied Mathematics and Computation, vol. 227, pp. 480-493, 2014.

[14] C. Li, X. Liao, and T. Huang, "Exponential stabilization of chaotic systems with delay by periodically intermittent control," Chaos, vol. 17, 2007.

[15] Y. Liang, X. Wang, and J. Eustace, "Adaptive synchronization in complex networks with non-delay and variable delay couplings via pinning control," Neurocomputing, vol. 123, pp. 292-298, 2014.

[16] Z. X. Liu, Z. Q. Chen, and Z. Z. Yuan, "Pinning control of weighted general complex dynamical networks with time delay," Physica A: Statistical Mechanics and Its Applications, vol. 375, no. 1, pp. 345-354, 2007.

[17] W. Yu, G. Chen, and J. Lü, "Synchronization via pinning control on general complex networks," SIAM Journal on Control and Optimization, vol. 51, no. 2, pp. 1395-1416, 2013.

[18] J. Zhou and J.-A. Lu, "Pinning adaptive synchronization of a general complex dynamical network," Automatica, vol. 44, no. 4, pp. 996-1003, 2008.

[19] J. Lü, X. Wu, W. Yu, M. Small, and J. Lu, "Pinning synchronization of delayed neural networks," Chaos, vol. 18, 2008.

[20] S. Cai, J. Hao, Q. He, and Z. Liu, "Exponential synchronization of complex delayed dynamical networks via pinning periodically intermittent control," Physics Letters A, vol. 375, no. 19, pp. 1965-1971, 2011.

[21] C. Zheng and J. Cao, "Robust synchronization of coupled neural networks with mixed delays and uncertain parameters by intermittent pinning control," Neurocomputing, vol. 141, pp. 153-159, 2014.

[22] S. Chen, G. Song, B.-C. Zheng, and T. Li, "Finite-time synchronization of coupled reaction-diffusion neural systems via intermittent control," Automatica, vol. 109, p. 108564, 2019.

[23] H. Li, "Synchronization of fractional-order complex dynamical networks via periodically intermittent pinning control," Chaos, Solitons and Fractals, vol. 103, pp. 357-363, 2017.

[24] Y. Xu, Q. Li, and W. Li, "Periodically intermittent discrete observation control for synchronization of fractional-order coupled systems," Communications in Nonlinear Science and Numerical Simulation, vol. 74, pp. 219-235, 2019.

[25] S. Boyd, L. El Ghaoui, E. Feron, and V. Balakrishnan, Linear Matrix Inequalities in System and Control Theory, SIAM, Philadelphia, PA, USA, 1994.

[26] J. Wu and L. Jiao, "Synchronization in complex delayed dynamical networks with nonsymmetric coupling," Physica A: Statistical Mechanics and Its Applications, vol. 386, no. 1, pp. 513-530, 2007. 